\title{
Specific Color Detection in Images using RGB Modelling in MATLAB
}

\author{
Vishesh Goel \\ Student \\ Department of \\ Computer Science \\ and Engineering, \\ Bharati Vidyapeeth's \\ College of Engineering, \\ Paschim Vihar, \\ New Delhi \\ Tarun Jain \\ Student \\ Department of \\ Computer Science \\ and Engineering, \\ Bharati Vidyapeeth's \\ College of Engineering, \\ Paschim Vihar, \\ New Delhi
}

\author{
Sahil Singhal \\ Student \\ Department of \\ Computer Science \\ and Engineering, \\ Bharati Vidyapeeth's \\ College of Engineering, \\ Paschim Vihar, \\ New Delhi \\ Silica Kole \\ Asst. Professor \\ Department of \\ Computer Science \\ and Engineering, \\ Bharati Vidyapeeth's \\ College of Engineering, \\ Paschim Vihar, \\ New Delhi
}

\begin{abstract}
This paper gives an approach to recognize colors in a twodimensional image using color thresh-holding technique in MATLAB with the help of RGB color model to detect a selected color by a user in an image. The methods involved for the detection of color in images are conversion of three dimensional RGB image into gray scale image and then subtracting the two images to get two dimensional black and white image, using median filter to filter out noisy pixels, using connected components labeling to detect connected regions in binary digital images and use of bounding box and its properties for calculating the metrics of each labeled region. Further the color of the pixels is recognized by analyzing the RGB values for each pixel present in the image. The algorithm is implemented using image processing toolbox in MATLAB. The results of this implementation can be used in security applications like spy robots, object tracking, segregation of objects based on their colors, intrusion detection.
\end{abstract}

\section{Keywords}

MATLAB, Image processing toolbox, color detection, RGB image, Image segmentation, Image filtering, Bounding box.

\section{INTRODUCTION}

Color is one of the most important characteristics of an image, if color in a live video or in a digital image can be detected, then the results of this detection can be used in various industrial and scientific applications. Color detection is the fundamental step in many computer vision systems. In this paper Image Processing toolbox in MATLAB is used for detection of a particular color in a given image. Image Processing Toolbox provides wide variety of referenced algorithms, methods and applications for image processing, visualization and segmentation. MATLAB based Image Processing [1][2][3] is well suited and most commonly used platform for implementation of an image based algorithm. An image is a matrix of pixel values. MATLAB takes every input as a matrix that is why, it is most commonly used platform for image processing. An image can be represented using many color models like gray-scale, RGB, HSV etc. Here RGB model is used to detect the colors in an image [4][5]. RGB model is a color model in which red, green and blue lights are added together in various ways to produce wide range of colors. In RGB model image is composed of a matrix of $\mathrm{MxNx} 3$ pixels with $\mathrm{M}$ rows and $\mathrm{N}$ columns of pixels for each red, green and blue color components of an image. The RGB model uses 8 bits for representation of red, green and blue color components of an image. Since in an RGB model image is represented in the form of a matrix, mathematical operations on the image can be applied to detect a particular color in an image.

Image Processing Toolbox is a MATLAB tool which provides vast variety of algorithms for image enhancement, analysis, object segregation, color detection, color conversion etc. It also provides the diverse support to image types which includes high dynamic range, tomographic and gigapixel resolution.

\section{METHODOLOGY}

To understand the basics of camera analyzing, one must know how a computer perceives an image. An image consists of a lot of pixels. Each pixel corresponds to a code.

The summation of these codes gives an entire image.

After the analyzation of these codes, these codes can be used for the definition of colors and these defined colors are used for pixel labelling which are used to recognize colors in the picture. 


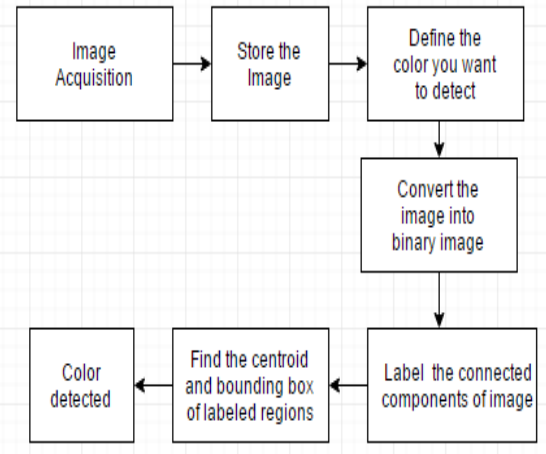

Fig 1. Flow Chart of algorithm

\section{IMPLEMENTATION OF ALGORITHM}

\subsection{Read the input image}

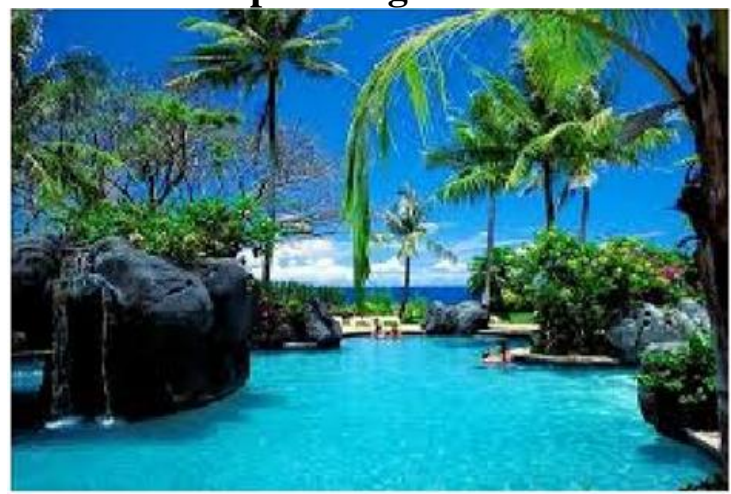

Fig 2. Input Image

The first stage of any image processing task is to have an image for detecting colors in it. One can capture it from the camera or load a previously clicked image from the memory.

Read the input image in RGB format which is the most commonly used format to represent colored images, if the resolution of the image is $\mathrm{MxN}$, then the RGB format of the image will be a three-dimensional matrix of size $\mathrm{MxNx} 3$ where each dimension of the matrix represents the red, green and blue color components of the image.

\subsection{Generating red, green and blue color bands of the image}

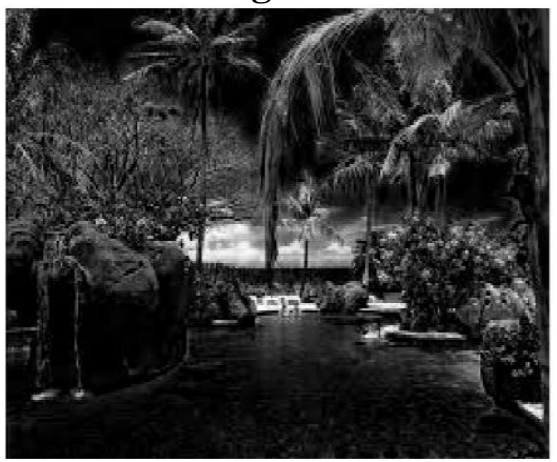

Fig 3. Red band Image

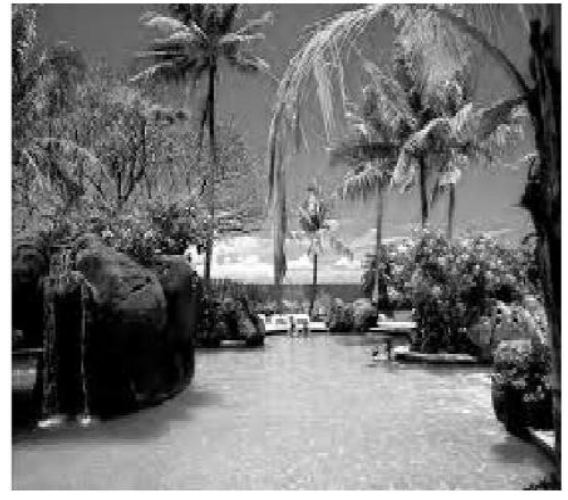

Fig 4. Green band Image

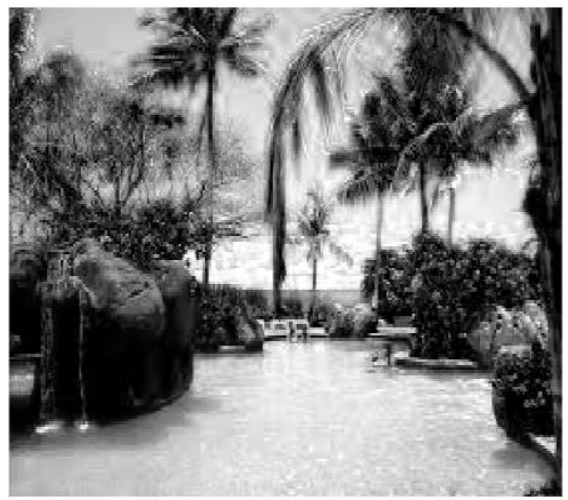

Fig 5. Blue band Image

Extract out the red, green and blue colour bands from the input image into three separate two dimensional matrices, one for each colour component. First, second and third slice of three dimensional matrices of RGB image contains the red, green and blue colour components respectively.

\subsection{Compute and plot the red, green and blue color band histogram}

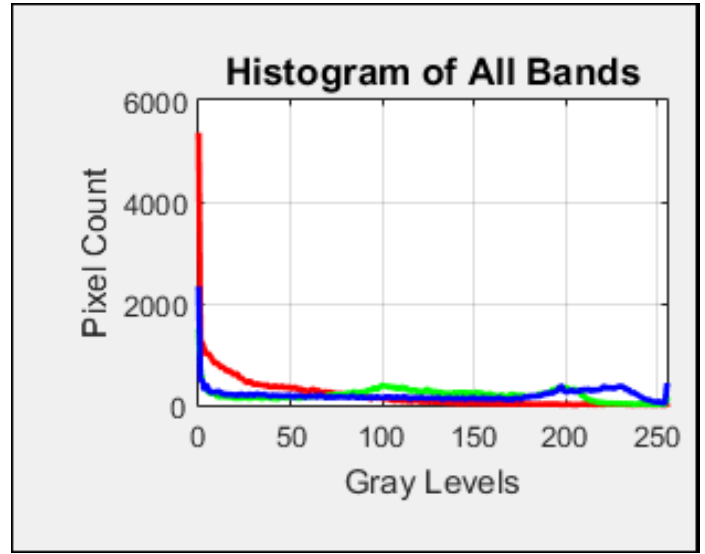

Fig 6. Histograms of all bands

In photography and image processing, histogram is the distribution of colors in an image. Compute and plot red, green and blue color band histogram using imhist() function of MATLAB. 


\subsection{Conversion of RGB image into grayscale and then to binary image using thresholding process}

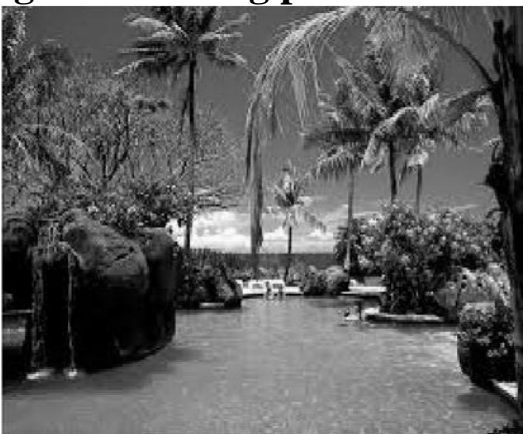

Fig 7. Gray scale Image

The RGB image is converted into grayscale image ${ }^{[6]}$, the grayscale format of the image will be a two-dimensional image containing the intensity value of each of the pixel of the image. Usually a grayscale image increases the speed of processing, ease of visualization, and reduces complexity of code by converting a three-dimensional image into twodimensional image resulting in reduction in number of bits used to represent each pixel of an image.

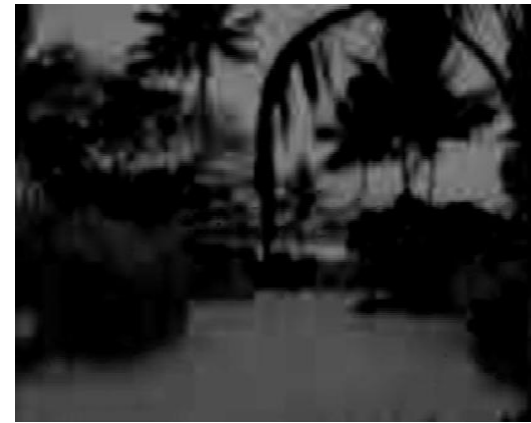

Fig 8. Subtracting gray scale Image from blue band image

This grayscale image is then subtracted from blue band image for the detection of blue color in the image. Similarly, for the detection of red and green colors, the grayscale image is subtracted from red band and green band respectively.

Subtraction of grayscale image from blue band of the input image is done to extract blue color components of the image.

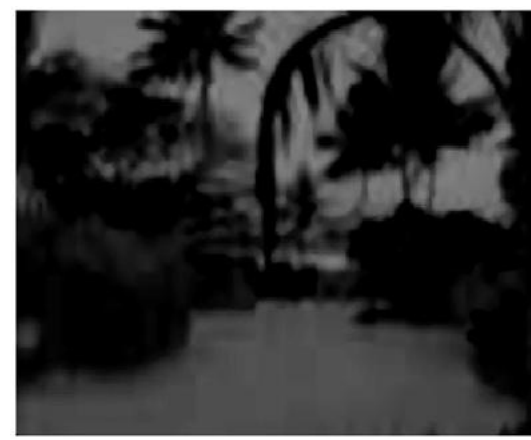

Fig 9. Image after median filtering

Median filtering is used to remove unwanted noise from the image while maintaining the originality of image $[15][16][17][18]$. In this process pixel value is converted into median value of $3 \times 3$ sampling window while keeping the pixel value of the border of sampling window unchanged.

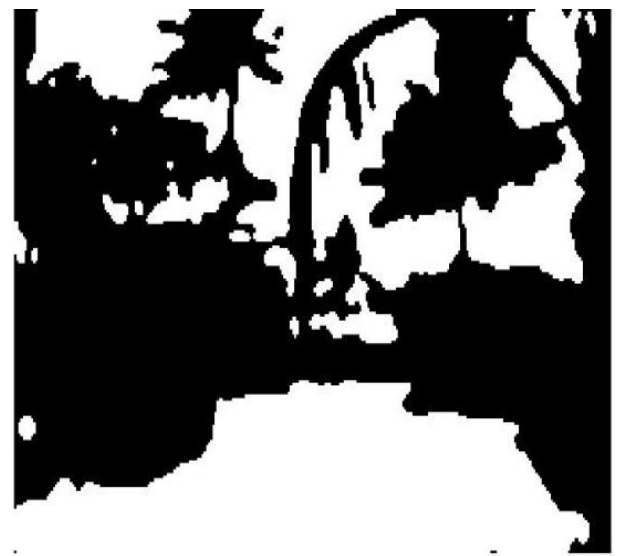

Fig 10. Binary image

This filtered image is then converted into binary image to work only on the area of interest ( 1 for yes and 0 for no) This binary image is generated from the filtered image using thresholding process ${ }^{[7][8][9][10]}$. In thresholding process each pixel of the image is given 1 or 0 on the basis that - if the pixel value is greater than the set threshold value then it is assigned ' 1 '(white) else ' 0 ' \{black). The threshold value of the image is calculated using the intensity histogram of the image $[11][12][13][14]$

\subsection{Removing objects having less than}

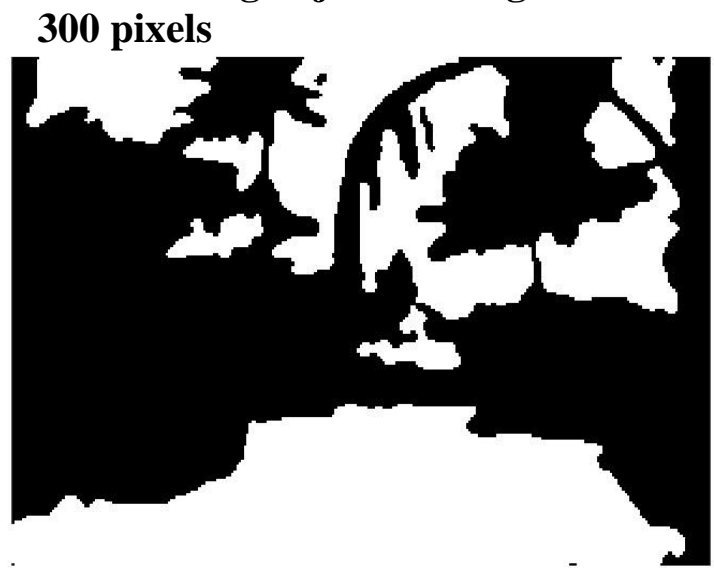

Fig 11. Image after removing objects having less than 300 pixels

All the objects of detected color having less than 300 pixels are removed from the image.

\subsection{Recognizing the boundaries of an object}

The $3 \mathrm{D}$ input image is already converted into $2 \mathrm{D}$ array. Now, any one out of the random pixel in object background interface is selected and is either moved in clockwise or anticlockwise direction to obtain other pixels. In this way, the boundary of the image is formed. Here boundaries of an object are searched diagonally(in 8 connected pixels) ${ }^{[19][20]}$. 


\subsection{Bounding box of the colored object}

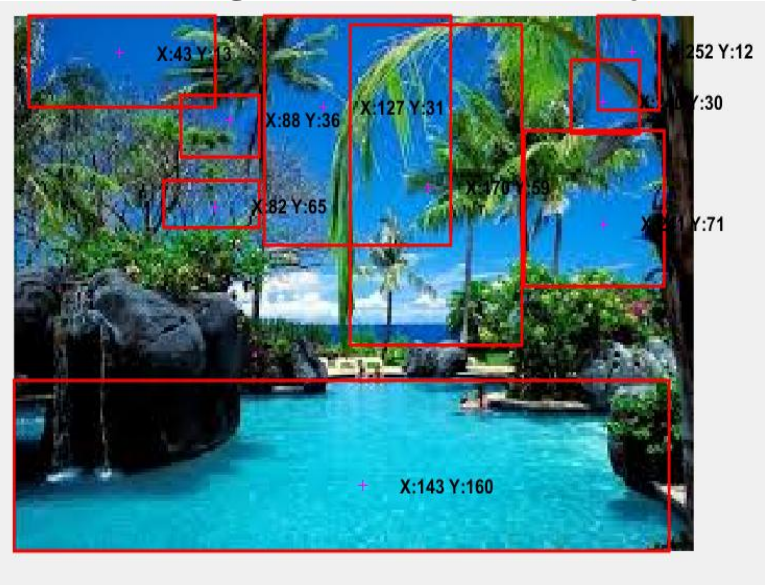

Fig 12. Bounding Box image

The detected object is shown through an imaginary rectangle with its sides parallel to the axes.

\section{RESULT}

A set of 100 images of different resolution and clarity were used for testing of this algorithm and the results were mostly accurate. Red, green, blue, magenta, cyan, yellow and white colors were successfully detected on these images.

The result of this detection depends on the threshold value that have been set for the images. The major problem with thresholding is that it considers only intensity values of the pixels and does not take into consideration any relationship between them. Sometimes extra pixels are detected which are not the part of the desired region, and with increase in noise these errors increases.

In the method of labeling of connected components for image segmentation there is one problem, that if overlapping objects are present in an image, then it will consider it as only one object.

\section{CONCLUSION}

With the help of image processing toolbox in the MATLAB, the program had been made which can detect red, blue, green, magenta, yellow, cyan colors. Also, the colored object is being enclosed inside a bounded region along with the centroid of that region.

\section{FUTURE SCOPE}

1. Computer vision- Color detection is the basic and important step for proceeding in computer vision. Some special type of spectacles can be made which will make use of computer vision (image processing) along with neural networks to provide an artificial vision to blind people.

2. Spy robots- The spy robots are made to identify objects in the place where they are launched. Object's shape, size, color, orientation is of importance to robot.

3. Object Segregation- An object can be segregated (separated) on the basis of its color.

4. Object Tracking- A moving object can be tracked on the basis of its color.

\section{REFERENCES}

[1] Rafael C. Gonzalez (University of Tennessee), Richard E. Woods (MedData Interactive) and Steven L. Eddins (The MathWorks, Inc.), in 'Digital Image Processing Using MATLAB' Second Edition,2009 by Gatesmark, LLC.

[2] Alasdair McAndrew, in 'An Introduction to Digital Image Processing with Matlab, Notes for SCM2511 Image Processing1', School of Computer Science and Mathematics, Victoria University of Technology.

[3] Digital image processing using Matlab -Gonzalez woods \& Eddins

[4] R. S. Berns, "Principles of Color Technology" (3rd edition New York: Wiley, 2000)

[5] G. Wyszecki and W. S. Styles, "Color Science: Concepts and Methods, Quantitative Data and Formulae" (2nd edition New York: Wiley, 1982)

[6] J. L. Vincent, "Morphological Grayscale Reconstruction in Image Analysis: Applications and Efficient Algorithms", IEEE Transactions on Image Processing, vol. 2, pp. 176-201, 1993.

[7] Devi, H.K.A., (2006). Thresholding: A Pixel-Level Image Processing Methodology Preprocessing Technique for an OCR System for the Brahmi Script. Ancient Asia. 1, pp.161-165.

[8] The Multi-stage Approach to Grey-Scale Image Thresholding for Specific Applications, Van Solihin and C. G. Leedham

[9] Document Image Analysis by Rangachar Kasturi, Louis Lam, Seong - Whan Lee \& Ching Y. Suen.

[10] M. Sezgin and B. Sankur, "Survey over Image Thresholding Techniques and Quantitative Performance Evaluation”, Journal of Electronic Imaging, vol. 13, no. 1, pp. 146-168, Jan. 2004.

[11] N. R. Pal and D. Bhandari, "On Object Background Classification", International Journal Syst. Science, vol. 23, no. 11, pp. 1903-1920, Nov. 1992.

[12] N. Otsu, "Threshold Selection Method from Gray-level Histograms", IEEE Transactions on Systems, Man, Cybernetics, vol. SMC-9, no. 1, pp. 62-66, Jan. 1979.

[13] J. Kittler and J. Illingworth, "Minimum error Thresholding", Pattern Recognition, vol. 19, no. 1, pp. 41-47, 1986.

[14] R. Guo and S. M. Pandit, "Automatic Threshold Selection based on Histogram Modes and a Discriminant Criterion", Machine Vision Applications, vol. 10, no. 5-6, pp. 331-338, Apr. 1998.

[15] Wang, Zhou, and David Zhang. "Progressive switching median filter for the removal of impulse noise from highly corrupted images." IEEE Transactions on Circuits and Systems II: Analog and Digital Signal Processing 46.1 (1999): 78-80.

[16] Brownrigg, D. R. K. "The weighted median filter." Communications of the ACM 27.8 (1984): 807 818. 
[17] Loupas, T., W. N. McDicken, and P. L. Allan. "An adaptive weighted median filter for speckle suppression in medical ultrasonic images." IEEE transactions on Circuits and Systems 36.1 (1989): 129-135.

[18] Eng, How-Lung, and Kai-Kuang Ma. "Noise adaptive soft-switching median filter." IEEE Transactions on image processing 10.2 (2001): 242-251.
[19] Haralick, Robert M., and Linda G. Shapiro. "Image segmentation techniques." Computer vision, graphics, and image processing 29.1 (1985): 100-132.

[20] F. Meyer, "Color image segmentation", Proceedings of 4th International Conference on Image Processing, pp. 523-548, 1992 